\title{
Ivosidenib Overview
}

\author{
Leila Abdullayeva* \\ Department of Clinical Anatomy and Operative Surgery, Kazakh National Medical University named after S D Asfendiyarov, Kazakhstan
}

*Corresponding author: Leila Abdullayeva, Department of Clinical Anatomy and Operative Surgery, Kazakh National Medical University named after S D Asfendiyarov, 94 Tole Bi Street Almaty, Kazakhstan.

To Cite This Article: Leila Abdullayeva, Ivosidenib Overview. 2020 - 8(1). AJBSR.MS.ID.001225. DOI: 10.34297/AJBSR.2020.08.001225.

Received: 制 February 24, 2020; Published: March 09, 2020

\begin{abstract}
Mutations of isocitrate dehydrogenase 1, observed in 7-14\% of patients with acute myeloid leukemia, have been associated with unfavorable prognosis. Ivosidenib (AG-120) is an oral, reversible small-molecule inhibitor of mutated IDH1 enzyme. This review provides a brief summary of ivosidenib monotherapy for patients with relapsed or refractory acute myeloid leukemia.
\end{abstract}

Keywords: Ivosidenib; Acute myeloid leukaemia; Differentiation syndrome

\section{Introduction}

Isocitrate dehydrogenase 1 (IDH1) is an enzyme that stimulates the oxidative decarboxylation of isocitrate to $\alpha$-ketoglutarate $(\alpha-K G)$. The mutation in IDH1 provokes uncontrollable cell proliferation and is recorded in $\sim 6 \%$ to $10 \%$ of patients with AML [1]. Ivosidenib (AG-120) is an oral, reversible small-molecule inhibitor of mutated IDH1 enzyme. In July of 2018, ivosidenib received the FDA approval for the treatment of refractory or relapsed AML (R/R AML) patients with IDH1 mutation [2]. The approval was established on results of a multicenter clinical trial with 179 patients. The Complete Response (CR) rate made up $24.7 \%$ while the Complete Response with partial hematologic improvement $(\mathrm{CRh})$ rate was $8 \%$.

\section{Ivosidenib Overview}

Ivosidenib is obtainable in pills with the dosage of $250 \mathrm{mg}$ under the brand name Tibsovo. The suggested dose is 500mg orally once a day [3]. Ivosidenib is rapidly absorbed (3 hours to peak plasma level) with cytochrome P450 3A4 (CYP3A4) being the primary metabolism route. $77 \%$ of the drug is excreted in the faeces without change and $17 \%$ in the urine (with $10 \%$ unaltered), presenting clearance volume of $4.3 \mathrm{~L} / \mathrm{hr}$ and an elimination half-life of 93 hours [4]. DiNardo et al. [5] reported a favourable clinical benefit of ivosidenib treatment among patients with relapsed or refractory AML. The CR rate composed $21.8 \%$ and the overall response rate was $39.1 \%$ [5].
Roboz et al. [6] in a phase 1 investigation of 34 patients with newly diagnosed AML unsuitable for regular therapy showed CR plus CRh rates of $42.4 \%$, and the median overall survival of 12.6 months [6]. General adverse effects of ivosidenib treatment include lethargy, arthralgia, diarrhoea, vomiting, fever, dyspnea, tussis, mucositis and dermatitis. Other occasional side effects involve differentiation syndrome, Guillian Barre' syndrome, QTc prolongation, and embryofetal toxicity [7]. Additionally, a rise in serum aminotransferase levels occurs in $15 \%$ to $20 \%$ of patients [3]. Differentiation syndrome combines symptoms of respiratory distress, hypoxia, pleural effusions, renal impairment, lymphadenopathy, peripheral oedema and weight gain. The start of the syndrome is within 2 to 8 weeks of treatment inception, and the management includes cessation of ivosidenib with the administration of corticosteroids and hydroxyurea in severe cases [8].

\section{Conclusion}

Ivosidenib monotherapy is an enthralling treatment option with positive response rates and notable clinical benefit in patients with newly diagnosed AML ineligible for conventional therapy. Its favourable toxicity profile, the simplicity of administration, and the potential for permanent remission make ivosidenib a strong therapeutic agent for the treatment of adult R/R AML with mutant IDH1. 


\section{References}

1. Patel KP, Ravandi F, Ma D, Paladugu A, Barkoh BA, et al. (2011) Acute myeloid leukemia with IDH1 or IDH2 mutation: frequency and clinicopathologic features. Am J Clin Pathol 135(1): 35-45.

2. Burgos PH, Patel J, Chen A (2019) Ivosidenib induction therapy complicated by myopericarditis and cardiogenic shock: A case report and literature review. J Oncol Pharm Pract 107815521986593.

3. (2012) LiverTox: Clinical and Research Information on Drug-Induced Liver Injury [Internet]. Bethesda (MD): National Institute of Diabetes and Digestive and Kidney Diseases, Ivosidenib.

4. Megías Vericat J E, Ballesta López O, Barragán E, Montesino P (2019) IDH1-mutated relapsed or refractory AML: current challenges and future prospects. Blood Lymphat Cancer 9: 19-32.

5. DiNardo CD, Stein EM, de Botton S, Gail J Roboz, Jessica K Altman, et al (2018) Durable remissions with ivosidenib in IDH1-mutated relapsed or refractory AML. N Engl J Med 378: 2386-2398.
6. Gail J Roboz, Courtney D DiNardo, Eytan M Stein, Stéphane de Botton, Alice S Mims, et al. (2019) Ivosidenib induces deep durable remissions in patients with newly diagnosed IDH1-mutant acute myeloid leukemia. Blood 135(7): 463-471.

7. Norsworthy KJ, Luo L, Hsu V, Gudi R, Dorff SE, et al. (2019) FDA Approval Summary: Ivosidenib for Relapsed or Refractory Acute Myeloid Leukemia with an Isocitrate Dehydrogenase-1 Mutation. Clin Cancer Res 25(11): 3205-3209.

8. Fathi AT, DiNardo CD, Kline I, Kenvin L, Gupta I, etal. (2018) Differentiation syndrome associated with enasidenib, a selective inhibitor of mutant isocitrate dehydrogenase 2: analysis of a phase 1/2 study. JAMA Oncol 4(8): 1106-1110. 